\title{
Getting lucky with adaptive optics: diffraction-limited resolution in the visible with current $A O$ systems on large and small telescopes
}

N. M. Law, R. G. Dekany, C. D. Mackay, A. M. Moore, M. C. Britton, et al.

N. M. Law, R. G. Dekany, C. D. Mackay, A. M. Moore, M. C. Britton, V. Velur, "Getting lucky with adaptive optics: diffraction-limited resolution in the visible with current AO systems on large and small telescopes," Proc. SPIE 7015, Adaptive Optics Systems, 70152I (10 July 2008); doi: 10.1117/12.788621

SPIE Event: SPIE Astronomical Telescopes + Instrumentation, 2008, Marseille, France 


\title{
Getting lucky with adaptive optics: diffraction-limited resolution in the visible with current $\mathrm{AO}$ systems on large and small telescopes
}

\author{
N.M. Law ${ }^{a}$, R. G. Dekany ${ }^{b}$, C.D. Mackay ${ }^{c}$, A.M. Moore ${ }^{b}$, M.C. Britton ${ }^{b}$ and V.Velur ${ }^{b}$ \\ ${ }^{a}$ Department of Astronomy, Mail Code 105-24, California Institute of Technology, 1200 East \\ California Blvd., Pasadena, CA 91125, USA; \\ ${ }^{b}$ Caltech Optical Observatories, Mail Code 105-24, California Institute of Technology, 1200 East \\ California Blvd., Pasadena, CA 91125, USA; \\ ${ }^{c}$ Institute of Astronomy, University of Cambridge, Madingley Road, Cambridge, CB3 OHA, UK;
}

\begin{abstract}
We have recently demonstrated diffraction-limited resolution imaging in the visible on the 5m Palomar Hale telescope. The new LAMP instrument is a Lucky Imaging backend camera for the Palomar AO system. Typical resolutions of $35-40$ mas with Strehls of $10-20 \%$ were achieved at $700 \mathrm{~nm}$, and at $500 \mathrm{~nm}$ the FWHM resolution was as small as 42 milliarcseconds. In this paper we discuss the capabilities and design challenges of such a system used with current and near future $\mathrm{AO}$ systems on a variety of telescopes. In particular, we describe the designs of two planned Lucky Imaging + AO instruments: a facility instrument for the Palomar 200" AO system and its PALM3K upgrade, and a visible-light imager for the CAMERA low-cost LGS AO system planned for the Palomar 60" telescope. We introduce a Monte Carlo simulation setup that reproduces the observed PSF variability behind an adaptive optics system, and apply it to predict the performance of 888Cam and CAMERA. CAMERA is predicted to achieve diffraction-limited resolution at wavelengths as short as $350 \mathrm{~nm}$. In addition to on-axis resolution improvements we discuss the results of frame selection with the aim of improving other image parameters such as isoplanatic patch sizes, showing that useful improvements in image quality can be made by Lucky $+\mathrm{AO}$ even with very temporally and spatially undersampled data.
\end{abstract}

\section{INTRODUCTION}

In recent work ${ }^{1}$ we demonstrated the first diffraction-limited direct imaging in the visible on large telescopes by combining Lucky Imaging frame selection and adaptive optics (hereafter Lucky+AO). Our new instrument, LAMP, obtained resolutions as good as 35 milliarcseconds at 700nm wavelength on the $5 \mathrm{~m}$ Palomar Hale telescope (figure 1). In this paper we evaluate the future possibilities of Lucky+AO instruments on current adaptive optics systems. In particular, we introduce simulation methods for Lucky+AO systems and predict the performance of two new instruments planned for telescopes at Palomar observatory.

In section 2 we briefly describe results from our Lucky+AO experiment and in section 3 we describe two new Lucky+AO instruments we are planning for the Palomar observatory. In section 4 we introduce a simulation method that successfully predicts many of the features of the PSF variability we have observed behind the Palomar adaptive optics system. We use our model to evaluate the performance of Lucky+AO systems in a variety of situations. Finally, section 5 investigates the uses of Lucky+AO systems for wider-field imaging without diffraction-limited resolution.

\section{RESULTS FROM THE LUCKY+AO EXPERIMENT ON THE PALOMAR 200”}

For an on-sky test of fast frame selection behind an adaptive optics system we designed and built an instrument for use at the Palomar 200" Hale telescope behind the PALMAO ${ }^{2,3}$ adaptive optics system. LAMP (LuckyCam, Aperture Masking and polarimetry at Palomar) was designed to offer several observation modes: a LuckyCam ${ }^{4}$ fast frame selection system, a visible-light aperture-masking mode and a high-angular-resolution high-precision polarimetry mode. In this section we describe the results from the LuckyCam fast-frame-selection mode; another paper in these proceedings describes results from the aperture masking mode.

Further author information: send correspondence to nlaw@ astro.caltech.edu

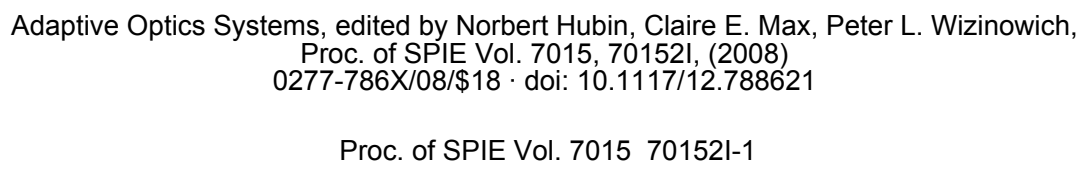




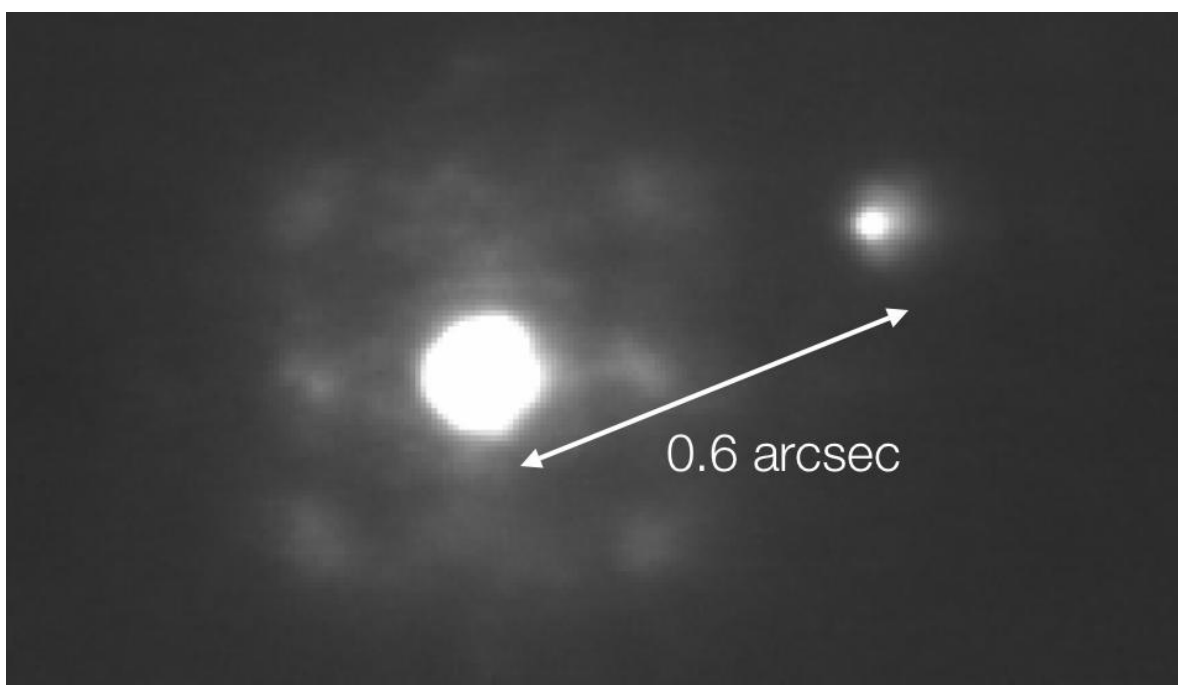

Figure 1. The binary HD 235089 ( $\Delta m \sim 4.5,0.57$ arcsec separation) observed at 710nm (10\% selection from 320 seconds at 50 FPS). The grid pattern in the PSF is due to uncorrected waffle modes from the AO system.

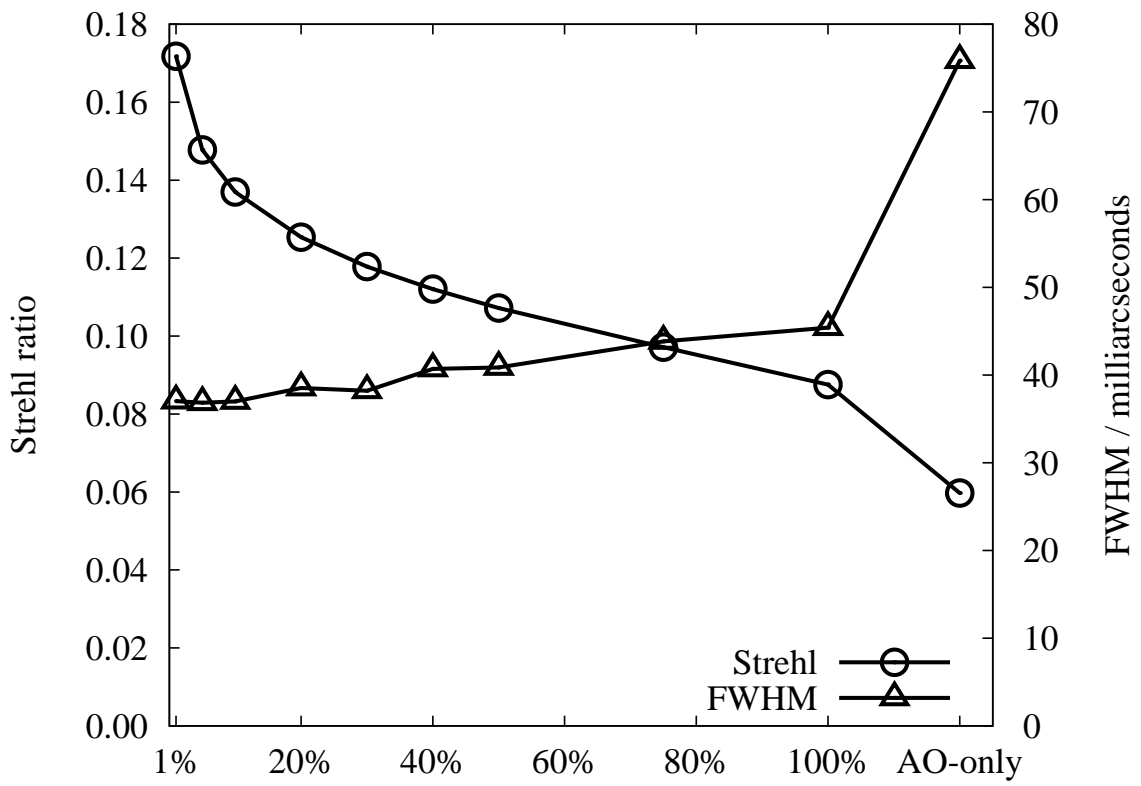

Figure 2. FWHM and Strehl ratio as a function of frame selection percentage for a typical LAMP run on HD 235089 in 0.86 arcsec seeing, taken at 50 frames per second in a $10 \mathrm{~nm}$ bandpass centered at $710 \mathrm{~nm}$.

The camera detector was based on an electron-multiplying 528×512 E2V CCD97. The CCD's electron-multiplication process allows detection of individual photons in each frame produced by the camera at the full quantum efficiency (up to $90 \%$ ) of the CCD. ${ }^{5}$ Our custom camera electronics were capable of running at up to 20 frames per second (FPS) in full 528x512 pixel frames; subarray readouts were used to increase the speed up to 50 FPS for some targets. The EMCCD gain was optimized for each target and ranged from 1 (no gain) to $\sim 10000$. The camera produced 14-bit data at 7.5 megapixels per second. The data were recorded using custom software; ${ }^{6}$ a lossless compression algorithm reduced the data transfer requirements by an average factor of 1.9 , allowing direct recording onto external USB hard disks.

We used the standard Lucky Imaging data reduction pipeline without modification; complete descriptions of the reduction process can be found in the literature. ${ }^{6,7}$ The data acquisition software was capable of displaying a realtime-preview of the Lucky Imaging output but the full data reduction was performed by a scripted process during daytime operations. 
Briefly, recorded frames were bias-corrected, flat-fielded and cosmic rays were removed. A bright star in the field was selected to serve as a Lucky Imaging guide star (in these observations this was typically the adaptive optics guide star). The frames were sorted in order of Strehl ratio and those that met a specific quality criterion were selected and aligned to produce a final high-angular-resolution image.

We estimate the Strehl ratio and optimal shift position for each image by cross-correlating the instantaneous guide star PSF with a diffraction-limited point spread function. The height of the peak of the resulting 2D array gives the degree of correlation $(\approx$ the Strehl ratio) and the position of the peak gives the optimal shift position to align the brightest speckles of each image. We perform the calculations on an image subsampled by $4 \times 4$ to allow sub-pixel image alignment using the Drizzle $^{8}$ algorithm.

The dispersion in Strehl ratios observed at $710 \mathrm{~nm}$ wavelength behind the AO system was high enough to give up to a factor of three improvement in both FWHM and Strehl ratio (figure 2). Figure 1 shows a typical LAMP observation; the FWHM resolution is $\sim 35$ milliarcseconds; the Strehl ratio is $\sim 0.15$. The Lucky+AO technique is a significant advantage over speckle imaging systems in that direct imaging is possible irrespective of the target's complexity.

A detailed evaluation of the Lucky+AO performance of LAMP is given in Law et al. 2008. ${ }^{1}$

\section{NEW PALOMAR INSTRUMENTS}

We plan two new instruments which will offer Lucky+AO capabilities as standard at Palomar.

\subsection{Cam}

888Cam is designed to be a permanent Lucky+AO instrument for the 5m Palomar Hale telescope. 888Cam will initially operate behind the current PALMAO system to pioneer visible light adaptive optics science on the $5 \mathrm{~m}$ telescope. Once the PALM3K high-order adaptive optics system is completed 888Cam will serve as a visible light imager offering both deep, high-Strehl imaging and fast frame rate imaging in the visible. 888Cam is based on LAMP, and is a simple re-imaging camera design. Off-the-shelf lenses reformat the $\mathrm{f} / 15$ beam from the PALMAO optics to provide a Nyquist-sampled 10 arcsecond field of view. An ADC based on the LAMP design will be operated in the collimated beam.

For reliability and cost reasons, $888 \mathrm{Cam}$ will offer a single fixed pixel scale which Nyquist samples the 35 milliarcsecond images produced in I-band by the $5 \mathrm{~m}$ telescope, and provides a 10 arcsecond field of view. Although small compared to many AO imagers, 10 arcseconds is comparable to the maximum radius over which we expect full PSF correction, and is limited by the need to Nyquist sample the PSFs when current commercially available EMCCDs have a maximum array size of $1024^{2}$ pixels. The 10 arcsecond field of view at very high angular resolution will complement the wider fields of view but lower resolutions available from Palomar's near-IR AO imager PHARO.

\subsection{Lucky+AO on CAMERA}

The CAMERA compact, autonomous MEMS-based Rayleigh Adaptive optics system (described in detail in an accompanying paper in these proceedings) is a robotic laser guide star adaptive optics system designed for the Palomar 60" telescope. The system offers dual band, diffraction limited imaging in the visible and near infrared over an arcminute field of view. The system will be deployed on the robotic $1.5 \mathrm{~m}$ telescope at Palomar Observatory and will operate autonomously to execute observations from a queue of targets and respond to transient alerts via VOEvent feeds. Use of several new technologies (MEMS deformable mirrors, ruggedized UV lasers, commodity computer wavefront reconstruction) will enable the construction of an LGS-AO system small and low-cost enough to operate efficiently on the Palomar 60-inch telescope.

CAMERA contains two science cameras; an IR camera operating from $950 \mathrm{~nm}$ to $1.6 \mu \mathrm{m}$ and a visible-light imager operating from $350 \mathrm{~nm}$ to $950 \mathrm{~nm}$. Both cameras have a fast-readout capability, with an EMCCD camera (i.e. a LuckyCam) in the visible-light role. In laser-guide-star mode CAMERA is thus able to use suitable tip/tilt guide stars in either the IR or the visible, with deep science imaging in the wavelength range not being used for tip/tilt sensing.

The visible-light LuckyCam imager component of CAMERA serves several roles:

1. Lucky+AO camera. CAMERA will provide diffraction-limited imaging down to $\sim 500 \mathrm{~nm}$ wavelengths in median Palomar seeing conditions (1.1 arcsec). The Lucky+AO capability of the system will allow diffraction-limited resolution to be attained in poorer conditions at much shorter wavelengths. 
2. Low-noise visible light imager. With Nyquist sampling at $600 \mathrm{~nm}$ and a 30 arcsec field of view, the EMCCD imager serves as the primary visible-light science camera for long-exposure deep imaging.

3. Fast visible tip/tilt sensor. Near-zero readout noise images from the EMCCD in tip/tilt sensing mode can be coadded to produce deep images of the region around the tip/tilt star. As many science targets are sufficiently bright to serve as their own guide stars, this provides CAMERA with a simultaneous dual-band imaging capability when visible-light tip/tilt guiding is performed. Using this imaging sensor for tip/tilt measurement allows centroiding using complex objects, for example a cluster of stars none of which is bright enough to guide on individually.

4. Backup Lucky Imaging system. Finally, the EMCCD camera can serve as a standard Lucky Imaging system, allowing operation on guide stars as faint as $\mathrm{m}_{\mathrm{I}}=15$ during conditions which prevent LGS propagation. Use of the AO system's deformable mirror to correct static aberrations will provide much better Lucky Imaging performance than is possible on most telescopes.

\section{PREDICTING THE PERFORMANCE OF LUCKY +AO SYSTEMS}

Variability in adaptive optics system performance is a result of complex interactions between the atmospheric turbulence and adaptive optics system. We here use two methods to predict the performance of Lucky+AO systems: scaling the results from LAMP (for situations within the LAMP parameter space) and Monte Carlo simulations of a simple adaptive optics system under a realistic distribution of Kolmogorov turbulence.

\subsection{Scaling from the LAMP results}

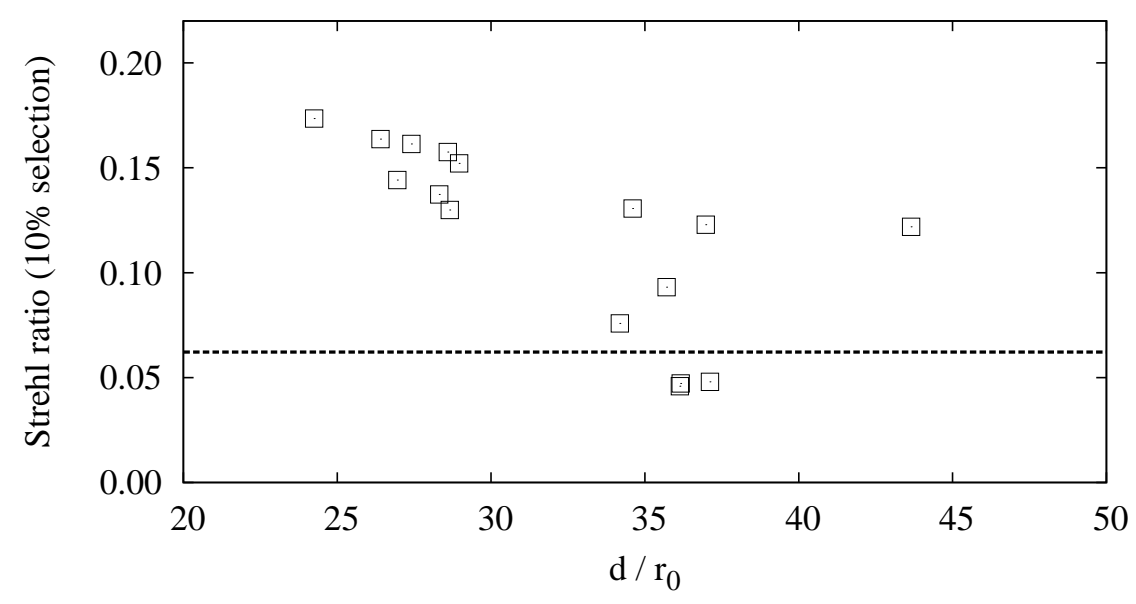

Figure 3. Strehl ratio as a function of $\mathrm{d} / \mathrm{r}_{0}$ - the results of Lucky Imaging of the AO data, selecting $10 \%$ of frames. The dashed line shows the average Strehl ratio achieved by the long-exposure AO imaging. To obtain a wide range of seeing the data presented here was taken with LAMP over several hours on two separate nights. Each datapoint is a 1.0-4.0 minute dataset taken at frame rates between 20 and 50 FPS. Data was recorded in $10 \mathrm{~nm}$ passbands centered at $670 \mathrm{~nm}$ or $710 \mathrm{~nm}$; the images were sampled at $14.9 \mathrm{mas} / \mathrm{pixel}$.

In figure 3 we show the Strehl ratio achieved by LAMP under a range of seeing values, in terms of $r_{0} / D$, the controlling parameter for the turbulence effect on image quality. Although there is some variance in the Strehl ratio achieved at any particular $r_{0} / D$, the combination of PALMAO and LAMP achieved moderate Strehl and diffraction-limited resolution in almost all encountered conditions (see Law et al. $2008^{1}$ for an extensive investigation of the performance of the system).

The performance given in figure 3 will be at least matched by $888 \mathrm{Cam}$, as the instrument is essentially a stripped-down version of LAMP. 
By scaling to $d / r_{0}$ we can start to predict the performance of a Lucky+AO system on different telescopes in a variety of conditions. We first note that many of our LAMP results were achieved at $d / r_{0}=35$, corresponding to median seeing $(\sim 1.0$ $\operatorname{arcsec})$ at Palomar on the $5 \mathrm{~m}$ telescope. This is equivalent to the same system operating on the $10 \mathrm{~m}$ Keck telescopes in 0.5 arcsec seeing at 700nm wavelengths, a seeing that is common at the Keck site (for example Racine et al. $1991{ }^{9}$ ).

At the other end of the size scale, the CAMERA Lucky+AO instrument, being on a $1.5 \mathrm{~m}$ telescope, would operate at $d / r_{0} \sim 15$ at $700 \mathrm{~nm}$ in 1.0 arcsec seeing. The adaptive optics system alone is expected to achieve $\geq 0.2 \mathrm{Strehl}$ ratios in this regime, without any need for frame selection (see the next section for a discussion of frame selection of moderate-Strehl images). However, in 2.0 arcsec seeing or when imaging in the blue $d / r_{0}$ may reach as high as $d / r_{0}=35$. In that case, Lucky+AO could be used to attempt to guarantee a particular Strehl ratio or resolution at the possible expense of losing some fraction of the exposure time to frame selection. This would greatly expand the wavelength range of the system, providing useful correction down to the blue sensitivity cutoff of the EMCCD at U-band. The ability to specify a particular Strehl ratio to be achieved may also be useful for the large surveys planned for the system, as it allows control of the system performance to achieve consistency between targets and reduce the performance dependence on turbulence conditions.

\subsection{Monte Carlo simulations}

The above results are indicative that Lucky $+\mathrm{AO}$ has a role to play on a variety of telescopes, but cannot be easily extrapolated to $d / r_{0}$ regimes not encountered. In addition, the argument is based on the assumption that $d / r_{0}$ is the single controlling parameter for the Strehl ratio variability behind an adaptive optics system. While $d / r_{0}$ is a good predictor of performance, as shown by figure 3, it is easy to think of effects that are not captured - for example, the different turbulence coherence times resulting from the different crossing times of different sized apertures in the same wind speed. To enable us to predict the performance of general Lucky+AO systems used in a variety of situations, we have tested Monte Carlo simulations of adaptive optics systems operating under realistic turbulence conditions.

Our models are based on the Arroyo adaptive-optics simulation code using a modified version of the standard singleconjugate adaptive optics simulation. ${ }^{10}$ We model the atmosphere as a set of six turbulent layers with total $r_{0}$ at the telescope equal to median Palomar seeing (1.1 arcsec) and a strength/height distribution typical for the Palomar observatory. The layers move with a Hardy wind model with typical wind speeds for Palomar.

The adaptive optics system is modeled with a $16 \times 16$ subaperture Shack-Hartmann sensor operating at $200 \mathrm{~Hz}$, a $16 \times 16$ actuator deformable mirror and a perfect detector with no non-common-path wavefront errors between it and the wavefront sensor (WFS). We assume that the DM and tip/tilt mirror respond instantaneously to the commands of the proportionalintegral (PI) controller, but use PI coefficients typical for the Palomar AO system. The effects of crosstalk between lenslets in the WFS are included in the simulations.

These simulations are designed to offer indications of the possible performance of the systems, but do not consider several effects which are likely to affect the temporal evolution of Strehl ratios:

1. We simulate an ideal adaptive optics system. The difficulties of real-world adaptive optics system implementation are likely to introduce increased Strehl ratio variability (e.g. Coud et al. $2000^{11}$ ). In particular, we do not simulate the effects of photon noise or readout noise in the WFS, we assume that all DM actuators are functional and are exactly identical, and we assume that the DM and tip/tilt mirror respond instantaneously and perfectly to the commands of a proportional-integral control system. We also simulate instantaneous images, so the image-smearing effect of imperfect tip/tilt correction is not included.

2. Intermittent seeing is not included. Baldwin, Warner \& Mackay ${ }^{12}$ have shown that, at least for some telescope sites, the mean seeing conditions can change on timescales (length scales in the turbulence layer) even shorter than a typical telescope crossing time. This may lead to rapid changes in the adaptive optics system performance required to reach a particular Strehl ratio, and will thus increase the image quality variability compared to that simulated.

3. Control loop gains. We do not optimize the control loop gains for different situations. The details of the Strehl ratio variability may be altered by changing the DM and tip/tilt control loop gains. We do not address this complex problem here, instead using the same gains for all simulations.

For these reasons the simulations should only be considered as one part of the possible PSF variability. Intermittent seeing and real AO systems will most likely introduce greater PSF variability than our simulated Kolmogorov turbulence and perfect AO. This extra variability may provide (short) periods of effectively improved seeing which Lucky+AO systems can take further advantage of. 

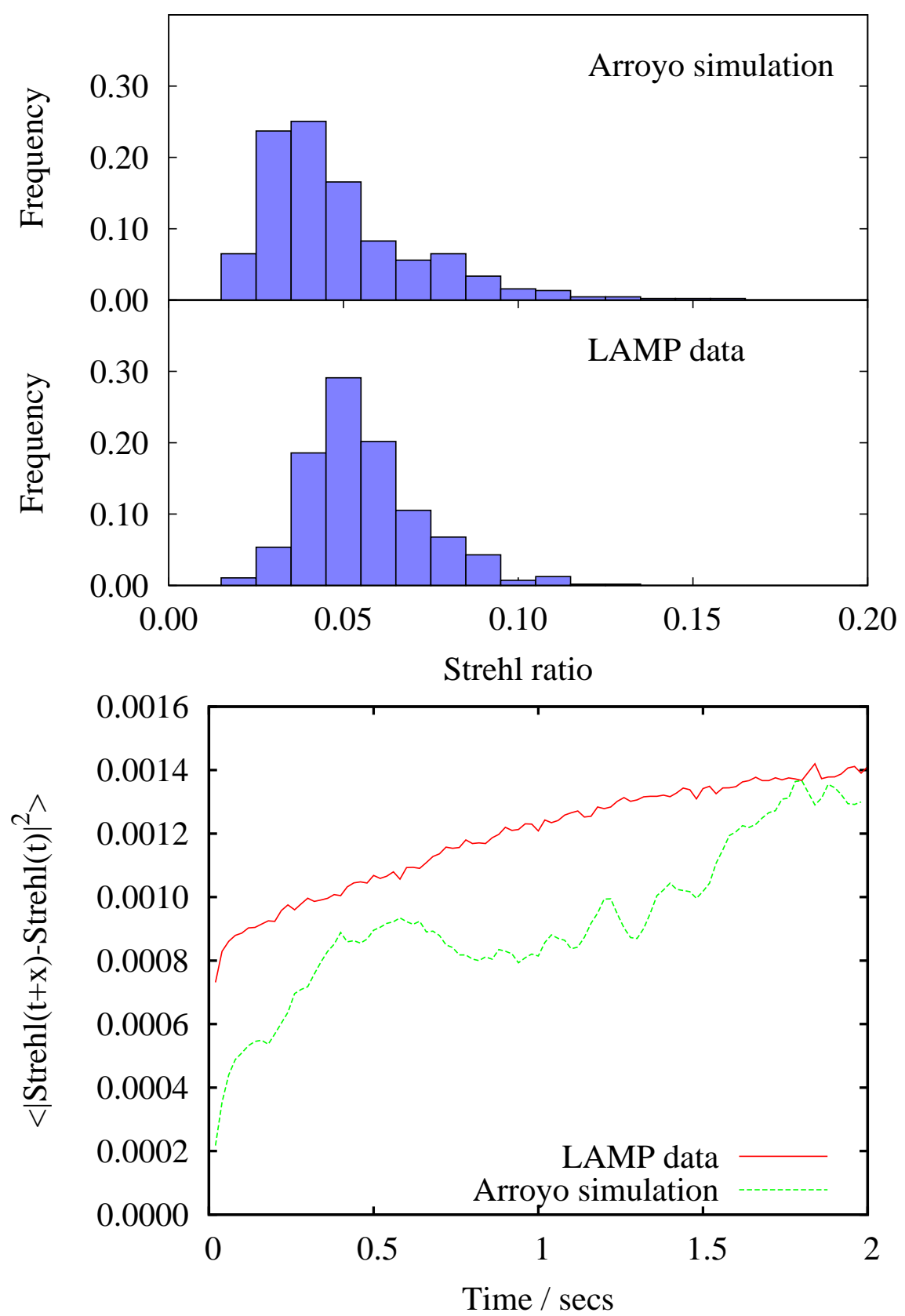

Figure 4. A comparison between LAMP observations and Arroyo simulations. We simulated the PALMAO adaptive optics system as described in the text, attempting to match the parameters of the LAMP runs shown in these figures: 1.0 arcsec seeing, 200 frames per second AO operation, 50 frames per second LAMP operation, 710nm detector wavelength.Top panel: comparing the distribution of Strehl ratios; Bottom panel: comparing the structure functions of the Strehl ratio changes. The small-scale features in the simulated structure functions are due to the relatively short simulation length.

\subsubsection{Results}

In figure 4 we compare the results of a simulated LAMP+PALMAO system on the Palomar 200" telescope to data recorded by the LAMP experiment. The agreement in Strehl ratio distributions is generally very good, with both the median Strehl ratio and the tail of high-Strehl frames reproduced well. The structure function of the Strehl ratios is a sensitive test of our simulations as it charts the temporal evolution of the Strehl ratio. Our simulations match the form of the structure function 
well, including the sharp drop at $1 / 25$ of a second and a good match to the power of the gradual rise in decorrelation out to several seconds, but appear to be missing a very fast timescale component of Strehl ratio variation seen in the real data. This cannot be due to photon noise - we chose a very bright $\left(m_{V}=6\right)$ star for these observations to ensure that photon noise is negligible. As the timescale of the variation is very fast compared to the wind crossing time for the turbulent layers, it is likely that the extra PSF variability is due to some unmodelled component of the adaptive optics system. The simulations thus under-predict the Strehl ratio variations on very short timescales, although the overall variability of the PSF is well-reproduced.

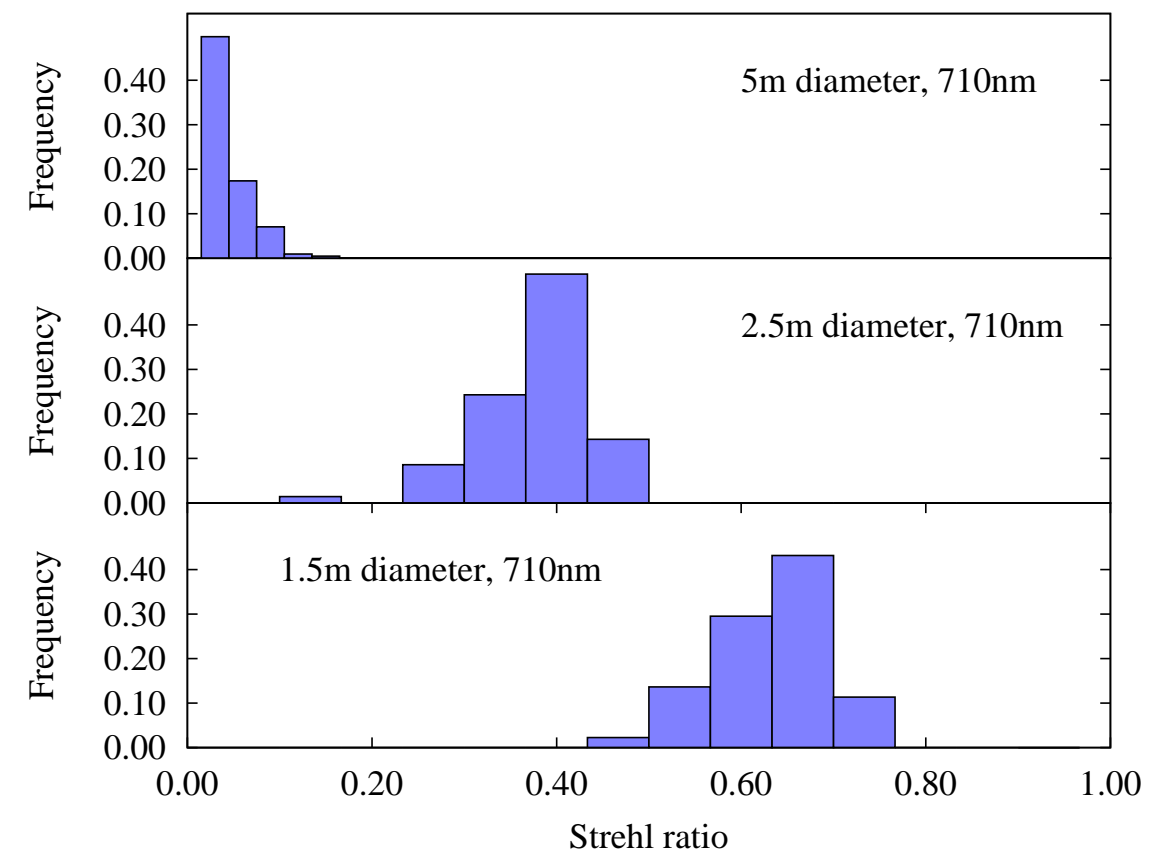

Figure 5. Simulated Strehl ratio histograms from simulations of moderate-order ( $16 \times 16$ subaperture) adaptive optics systems on $1.5 \mathrm{~m}$, $2.5 \mathrm{~m}$ and $5 \mathrm{~m}$ telescopes. All histograms are for $1.0 \mathrm{arcsec}$ seeing at $710 \mathrm{~nm}$.

In figure 5 we investigate the change in Strehl ratio distribution with telescope size. Smaller apertures show much better adaptive optics performance, as expected. For Lucky Imaging applications, the most interesting feature is a transition between positively-skewed Strehl histograms (for which higher Strehl outliers are common) and negatively skewed histograms as the system performance increases. In the low-Strehl regime (top panel) we expect to be able to achieve Strehl ratio increases of factors of two or more by frame selection, but for smaller telescopes with AO systems already giving moderate Strehl images in the visible we expect to only produce small increases in Strehl ratio. Generally, frame selection appears to be most useful to lift poor Strehl data into the moderate-Strehl regime, but is not useful to further improve data already in that regime.

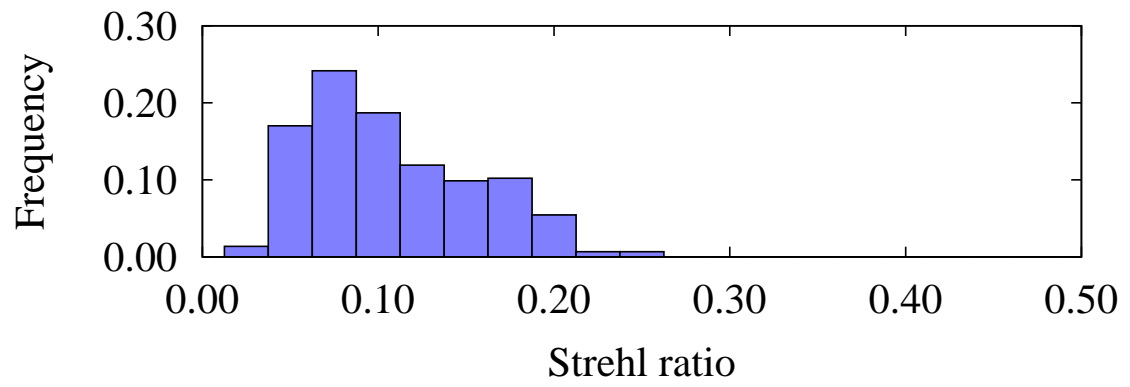

Figure 6. A simulated Strehl ratio histogram for the CAMERA adaptive optics system operating at $350 \mathrm{~nm}$ in median Palomar conditions.

As discussed above, when operating in the blue or in poor seeing, the CAMERA system may reach $d / r_{0}$ as high as 
35, similar to the LAMP conditions. Our simulations agree that in those conditions there will be significant gains to be made from frame selection (figure 6). Using Lucky+AO to achieve Strehl ratios in excess of 0.10 (up to 0.20 for stringent frame quality criteria), the CAMERA system may be able to achieve fully diffraction-limited imaging at $350 \mathrm{~nm}$. The 0.06 arcsec resolution at $350 \mathrm{~nm}$ complements the similar resolution achievable with PALMAO on the 200-inch Hale telescope in J-band, or in K-band on Keck.

Our simulated Strehl ratio histograms are in agreement with the results of Gladysz et al. $2008{ }^{13}$ where the histograms of instantaneous Strehl ratios in various turbulence parameters are calculated analytically. Our simulations model the full effects of a real AO system, including the effects of operating the correction mirrors behind a proportional-integral controller and Shack-Hartmann wavefront sensor, and so provide an independent confirmation to the Gladysz results.

\section{A DIFFERENT REGIME: NON-DIFFRACTION-LIMITED OPERATION}

In the above sections we have investigated the use of Lucky+AO to attain diffraction-limited resolution at shorter wavelengths than possible with current adaptive optics systems alone. Diffraction-limited resolution requires a PSF which is properly sampled in both space and, as we show in Law et al. $2008,{ }^{1}$ attaining the best resolutions requires a fast frame rate of 30+ frames per second.

With LAMP we undertook several experiments to investigate if scientifically useful performance can still be attained with spatially and temporally undersampled data. We observed the core of the M13 globular cluster in SDSS i'-band with LAMP's largest field of view: 60 milliarcsecond pixels and full frame imaging giving a frame rate of 20 FPS. These observations are $\sim 6 \times$ undersampled spatially and about $2.5 \times$ undersampled temporally. The observations were undertaken in 0.60 arcsec seeing and the dataset included 6000 frames taken over 5 minutes.

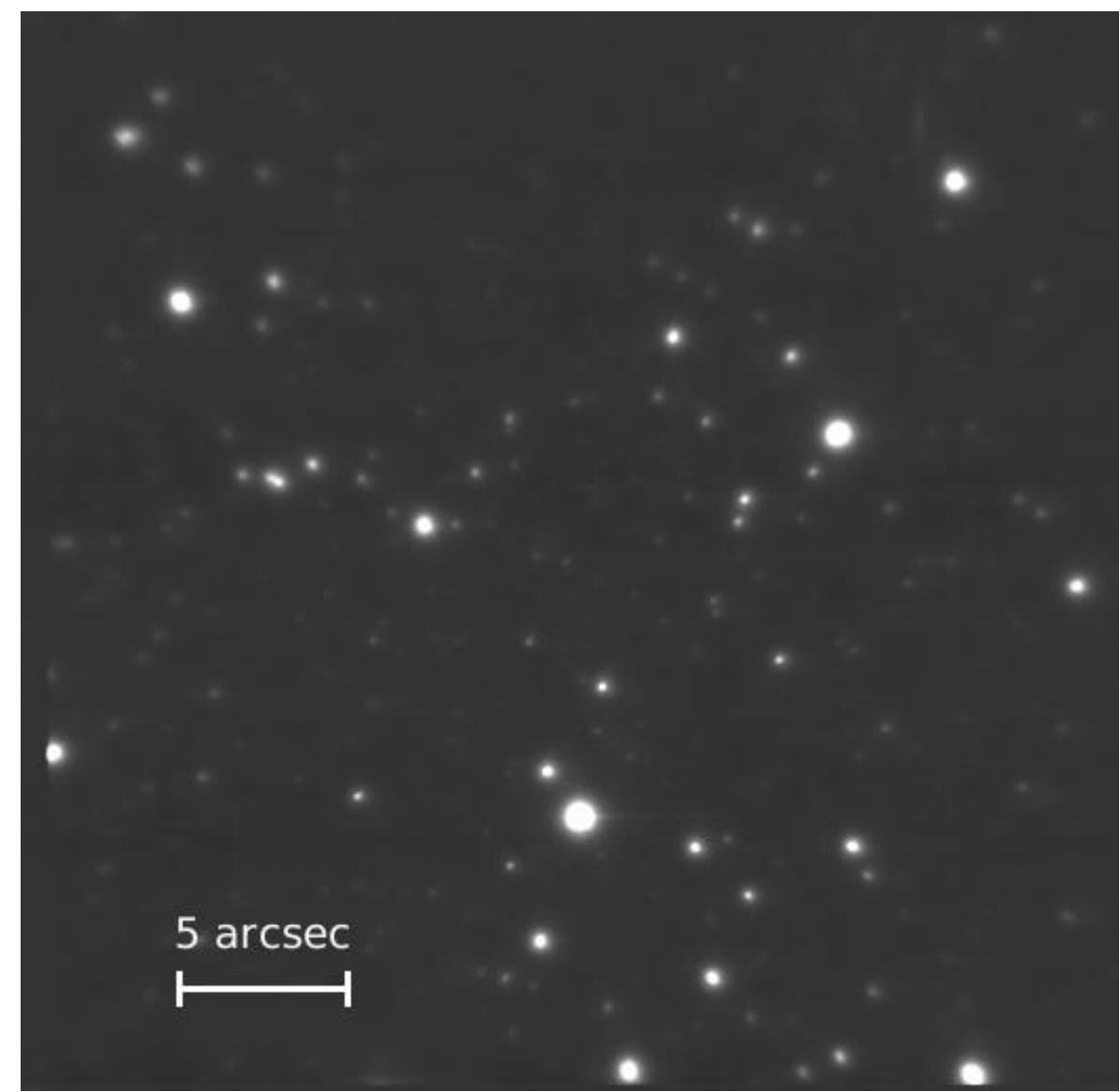

Figure 7. A $20 \%$ selected Lucky+AO view of the center of the M13 globular cluster, taken with taken with severely undersampled pixels in the SDSS i' filter (770 $\mathrm{nm}$ central wavelength). 
In figure 7 we show the Lucky+AO output image from a $20 \%$ selection from our dataset; figure 8 maps the PSF FWHM variation across the field of view. The FWHM resolution is improved by a factor of $\sim 3 \times$ to 200 milliarcsecond FWHM in the vicinity of the guide star, and the Lucky+AO FWHM resolution is improved by about 100 milliarcseconds across the field compared to the long-exposure AO imaging. At the edge of our field the AO-only PSF FWHM is approaching the seeing-limited 0.6 arcsec value, but the Lucky+AO image is has FWHM approximately half the seeing-limited value. This suggests that the useful field of view* may be somewhat larger for Lucky+AO systems than straight AO imaging. This is similar to without-AO Lucky Imaging results, ${ }^{1,12}$ where frame selection (without AO) is suggested to lead to periods of greater isoplanatic patch size.
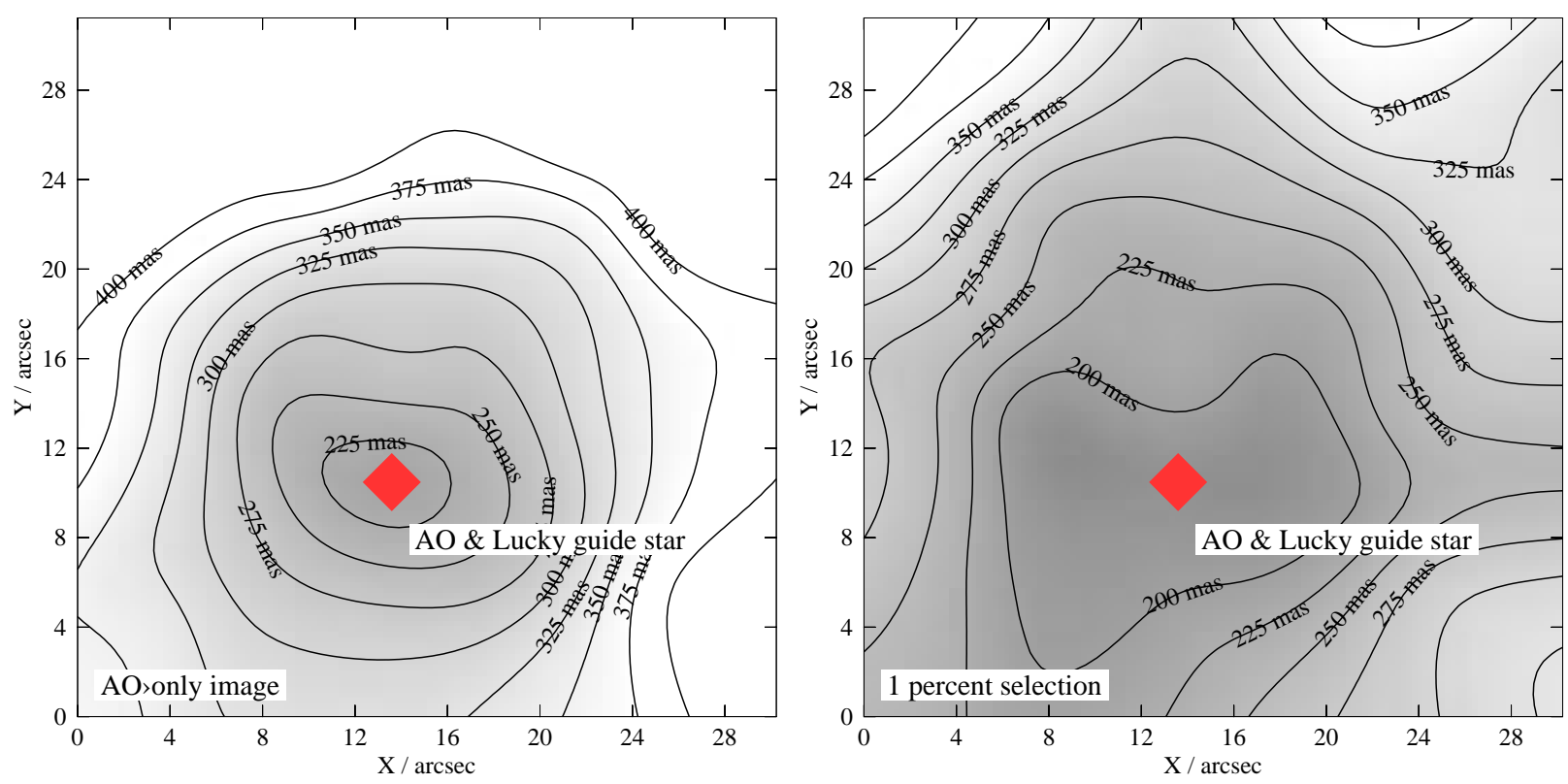

Figure 8. Maps of the PSF FWHM across the field shown in figure 7, interpolated from the measured FWHMs of 74 stars distributed throughout the field.

In Law et al. $2008^{1}$ we investigate the possibilities of using multiple Lucky Imaging guide stars and a single AO guide star to further improve the useful field of view of the system, finding that the area of sky improved to sub-300 milliarcsecond resolution can be increased by at least a factor of 4 .

\section{CONCLUSIONS}

We have shown that the combination of Lucky Imaging and Adaptive Optics can provide substantial performance increases in conditions where an adaptive optics system alone provides poor or very poor correction. We introduced a Monte Carlo simulation setup that reproduces most of the observed PSF variability behind an adaptive optics system. We apply the simulations to predict that the CAMERA adaptive optics system for the Palomar 60-inch telescope will be able to produce diffraction-limited moderate-Strehl imaging at wavelengths as short as $350 \mathrm{~nm}$ using Lucky+AO. Generally, on large telescopes in typical conditions, Lucky+AO enables diffraction-limited performance in the visible with current moderateorder adaptive optics systems.

\section{ACKNOWLEDGMENTS}

We particularly thank the PALMAO team, especially Jenny Roberts and Antonin Bouchez, for all their help during the design, setup and operation of the LAMP instrument. Thanks also go to the Palomar Observatory team for great assistance throughout our run.

${ }^{*}$ we do not use isoplanatic patch here because of the severely undersampled data and non-diffraction-limited resolution. 


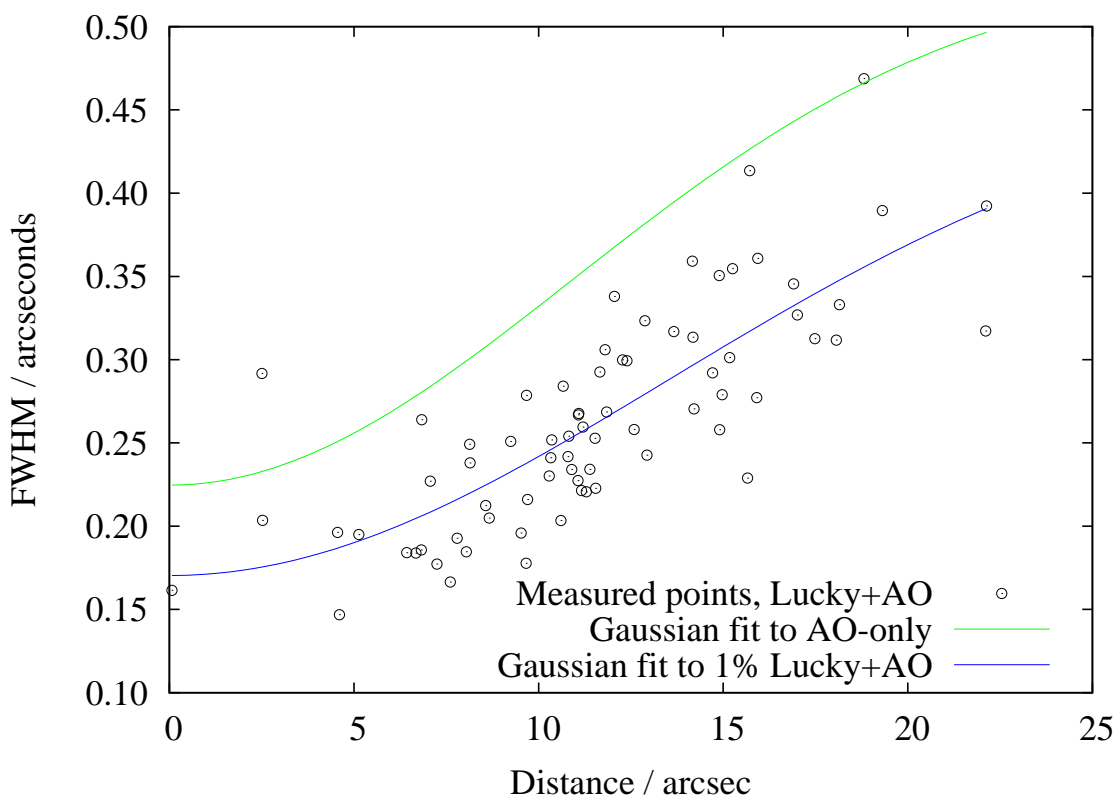

Figure 9. The falloff in image resolution as a function of distance for severely undersampled data taken in i'-band with LAMP. Gaussian fits to the falloff in image resolution for both AO-only and $1 \%$ Lucky+AO selected data are shown. To avoid confusion, we only show the measured points only for the $1 \%$ Lucky+AO selection image.

\section{REFERENCES}

[1] Law, N. M., Mackay, C. D., Dekany, R. G., Ireland, M., Lloyd, J. P., Moore, A. M., Robertson, J. G., Tuthill, P., and Woodruff, H., "Getting Lucky with Adaptive Optics: Fast AO Image Selection in the Visible with a Large Telescope," ArXiv e-prints, submitted to ApJ $\mathbf{8 0 5}$ (May 2008).

[2] Troy, M., Dekany, R. G., Brack, G., Oppenheimer, B. R., Bloemhof, E. E., Trinh, T., Dekens, F. G., Shi, F., Hayward, T. L., and Brandl, B., "Palomar adaptive optics project: status and performance," in [Proc. SPIE Vol. 4007, p. 31-40, Adaptive Optical Systems Technology, Peter L. Wizinowich; Ed.], Wizinowich, P. L., ed., Presented at the Society of Photo-Optical Instrumentation Engineers (SPIE) Conference 4007, 31-40 (July 2000).

[3] Dekany, R. G., Brack, G., Palmer, D., Oppenheimer, B. R., Hayward, T. L., and Brandl, B., "First tip-tilt correction with the Palomar 200-in. adaptive optics system," in [Proc. SPIE Vol. 3353, p. 56-59, Adaptive Optical System Technologies, Domenico Bonaccini; Robert K. Tyson; Eds. ], Bonaccini, D. and Tyson, R. K., eds., Presented at the Society of Photo-Optical Instrumentation Engineers (SPIE) Conference 3353, 56-59 (Sept. 1998).

[4] Mackay, C. D., Baldwin, J., Law, N., and Warner, P., "High-resolution imaging in the visible from the ground without adaptive optics: new techniques and results," in [Proceedings of the SPIE, Volume 5492, pp. 128-135 (2004). ], 128135 (Sept. 2004).

[5] Mackay, C., Basden, A., and Bridgeland, M., "Astronomical imaging with L3CCDs: detector performance and highspeed controller design," in [Proceedings of the SPIE, Volume 5499, pp. 203-209 (2004). ], 203-209 (Sept. 2004).

[6] Law, N. M., "Lucky imaging: diffraction-limited astronomy from the ground in the visible," The Observatory $\mathbf{1 2 7}$, 71-71 (Feb. 2007).

[7] Law, N. M., Mackay, C. D., and Baldwin, J. E., "Lucky imaging: high angular resolution imaging in the visible from the ground," AEA 446, 739-745 (Feb. 2006).

[8] Fruchter, A. S. and Hook, R. N., "Drizzle: A Method for the Linear Reconstruction of Undersampled Images," PASP 114, 144-152 (Feb. 2002).

[9] Racine, R., Salmon, D., Cowley, D., and Sovka, J., "Mirror, dome, and natural seeing at CFHT," PASP 103, 10201032 (Sept. 1991).

[10] Britton, M. C., "Arroyo," in [Modeling and Systems Engineering for Astronomy. Edited by Craig, Simon C.; Cullum, Martin J. Proceedings of the SPIE, Volume 5497, pp. 290-300 (2004). ], Craig, S. C. and Cullum, M. J., eds., Presented at the Society of Photo-Optical Instrumentation Engineers (SPIE) Conference 5497, 290-300 (Sept. 2004). 
[11] Coudé du Foresto, V., Faucherre, M., Hubin, N., and Gitton, P., "Using single-mode fibers to monitor fast Strehl ratio fluctuations. Application to a $3.6 \mathrm{~m}$ telescope corrected by adaptive optics," AAPS 145, 305-310 (Aug. 2000).

[12] Baldwin, J. E., Warner, P. J., and Mackay, C. D., "The point spread function in Lucky Imaging and variations in seeing on short timescales," AEA 480, 589-597 (Mar. 2008).

[13] Gladysz, S., Christou, J. C., Bradford, L. W., and Roberts, Jr, L. C., "Temporal variability and statistics of the Strehl ratio in adaptive-optics images," ArXiv e-prints 801 (Jan. 2008). 